\section{Programa de tamizaje universal e intervención precoz (PTUIP) en hipoacusia sensorioneural bilateral congénita. Tarea pendiente desde la perspectiva de políticas públicas de salud en Chile}

\author{
NICOLÁS ALBERTZ ${ }^{\mathrm{a}}$, FELIPE CARDEMIL ${ }^{1, \mathrm{~b}}$, MARITZA RAHAL $^{1}$, \\ FRANCISCA MANSILLA, ${ }^{1, \mathrm{c}}$, RODRIGO CÁRDENAS ${ }^{1, \mathrm{c}}$, PEDRO ZITKO ${ }^{2, \mathrm{~d}}$

\section{Universal screening program and early sensorineural hearing loss in Chile} \\ intervention (USPEI) in congenital bilateral
}

Congenital hearing loss is the total or partial inability to hear sounds through the ears. It is the most common disability in newborns in Chile and worldwide, and is a permanent condition. The direct impact on children who are not adequately diagnosed is the alteration in acquisition of language and cognitive skills and a decline in their social and school insertion, jeopardizing their professional and potentially productive life. Universal screening programs for hearing loss are essential for the diagnosis, since $50 \%$ of infants with hearing loss have no known risk factor. Screening before one month of age, confirmation before 3 months, and effective intervention before 6 months, allows the development of these children as if they had normal hearing. In Chile there is a selective program of screening for infants aged less than 32 weeks or 1,500 grams, as part of Explicit Health Guarantees, but it covers only $0.9 \%$ of newborns per year. Therefore, a large majority of children remain without diagnosis. The aim of this review is to compare the situation in Chile with other countries, raising the need to move towards a universal neonatal hearing loss screening program, and propose necessary conditions in terms of justification and implementation of a universal screening public policy.

(Rev Med Chile 2013; 141: 1057-1063).

Key words: Health policy; Hearing loss; Neonatal screening.
${ }^{1}$ Servicio de

Otorrinolaringología,

Hospital Barros Luco

Trudeau, Santiago, Chile.

${ }^{2}$ Unidad de Estudios

Asistenciales, Hospital

Barros Luco Trudeau,

Santiago Chile.

aMagíster (c) en Salud

Pública Universidad de

Chile, Santiago, Chile,

Becario CONICYT.

bPrograma de Doctorado

en Salud Pública

Universidad de Chile,

Santiago, Chile, Becario

CONICYT.

'Fonoaudiólogo

${ }^{\mathrm{d}}$ Magíster en

Epidemiología.

El presente estudio se realizó sin ningún tipo de apoyo financiero, ni presenta conflictos de interés.

Recibido el 9 de octubre de 2012, aceptado el 1 de mayo de 2013.

Correspondencia a: Nicolás Albertz Las Verbenas 9000 , Depto. 1105, Las Condes, Santiago, Chile. E-mail:nalbertz@ ug.uchile.cl

\section{Definición y relevancia del problema}

L a hipoacusia sensorioneural bilateral congénita (HSNBC) corresponde a la discapacidad más frecuente del recién nacido. Tiene un carácter permanente e irreversible y actualmente persiste como un problema de salud pública pendiente por resolver en Chile ${ }^{1}$. Consiste en la incapacidad total o parcial de percibir sonidos, secundario a una alteración o disfuncionalidad a nivel de los receptores auditivos en el oído interno, o bien a la alteración de la vía auditiva nerviosa en cualquiera de sus niveles hasta llegar a la corteza cerebral $^{2,3}$.

Las consecuencias directas en aquellos niños que no son pesquisados e implementados adecuadamente son la generación de alteraciones en la adquisición del lenguaje y cognitivas, dis- 
minuyendo su inserción social y escolar y, a su vez, comprometiendo su potencial profesional y productivo a lo largo de toda la vida ${ }^{4-7}$. Por su parte, los objetivos de los programas de pesquisa precoz de hipoacusia consisten en el tamizaje, diagnóstico e implementación de los afectados con el fin de optimizar la comunicación, los resultados sociales, académicos y profesionales para cada niño con pérdida auditiva, habiéndose demostrado que la detección antes de 3 meses de vida, acompañada de una intervención efectiva antes de los 6 meses, permite el desarrollo de estos niños tal como si tuviesen una audición normal, resultado que se incrementaría mientras más temprano se realice el diagnóstico y tratamiento ${ }^{4,5,7,8}$.

\section{Presentación clínica e historia natural}

Cincuenta por ciento de los casos corresponden a recién nacidos sanos sin factores de riesgo, presentándose de manera típica como un recién nacido de aspecto saludable sin que existan elementos que orienten a su presencia o generen algún grado de sospecha $a^{9}$. Por este motivo la mayoría de las veces la hipoacusia pasa desapercibida al momento de nacer o en los primeros controles sanos.

La sospecha recién aparece luego de varios meses o incluso algunos años, cuando los padres notan que sus hijos no han superado hitos del desarrollo del lenguaje o no han aprendido a hablar. Esto lleva a que muchos casos de hipoacusia moderada se detecten recién en la edad escolar ${ }^{10}$, sospechada por profesores basados en un bajo rendimiento académico, siendo muchas veces confundida con déficit atencional, retraso mental, autismo u otros diagnósticos neurológicos. Este hecho ha sido corroborado al evidenciarse que antes de la implementación de programas de pesquisa precoz de hipoacusia, la edad promedio de diagnóstico era entre los 2 y 3 años, para reducirse a los 5 a 7 meses con su implementación ${ }^{1,11,12}$.

El manejo incluye el uso de audífonos para casos de hipoacusia en grado leve y moderado, e implantes cocleares para severa y profunda, con algunas excepciones. A esto hay que sumar las horas de terapeutas de lenguaje, médico, psicólogo e insumos clínicos y operatorios, entre otros. Pese a ello, en series de Estados Unidos de Norteamérica se ha visto que un niño correctamente implementado, que logre desarrollar audición que le permita escuchar, podrá aprender como un niño sin hipoacusia ${ }^{13}$, requerirá menos horas de educación especial ${ }^{14}$, mejorará su calidad de vida ${ }^{15}$, y se infiere que a largo plazo se podrá desarrollar como una persona sin discapacidades, lo que tiene un impacto en menores costos al país y mayor productividad $^{14}$.

\section{Epidemiología}

Múltiples estudios han reportado cifras entre 1 y 5 casos cada 1.000 nacidos vivos en sus distintos grados de severidad ${ }^{1,16-18}$. En Chile no se tienen estimaciones certeras respecto de la incidencia, ni del total de niños con hipoacusia. Sin embargo, en base a la encuesta CASEN 2009, se estima que cerca de 25 mil personas viven actualmente en Chile con hipoacusia desde el nacimiento ${ }^{19}$. Una serie de Clínica Las Condes, institución que realiza tamizaje universal, reportó una incidencia de 2,8 casos cada 1.000 nacidos vivos con un diagnóstico a los 3 meses de $88,5 \%{ }^{20}$. Tomando como base 250.000 nacimientos anuales en nuestro país, se puede plantear que nacen actualmente en Chile hasta 1.250 niños con hipoacusia, de los cuales un porcentaje importante no son oportunamente diagnosticados ni habilitados.

\section{Programas de pesquisa en el mundo}

Al comparar la situación local con la de países de la región, se aprecian situaciones disimiles: Argentina posee un programa de tamizaje universal implementado desde 1997, con legislación vigente desde 2001 que establece el derecho a recibir tratamiento adecuado desde $2005^{21}$; Brasil posee un sistema que cubre $4 \%$ de las maternidades ${ }^{22}$; Colombia posee una legislación referente a la pesquisa universal desde 2005, que actualmente se encuentra en implementación ${ }^{16}$; Puerto Rico es el único otro país además de Argentina que posee tamizaje universal de hipoacusia neonatal ${ }^{16}$.

En otras regiones, como en Estados Unidos de Norteamérica, el año 1993 el National Institutes of Health realizó un consenso en que se impulsó el tamizaje universal ${ }^{23}$. Posteriormente, el Joint Committee on Infant Hearing ha elaborado posiciones oficiales de manera de reglamentar y evaluar la estrategia ${ }^{4,24}$. China también posee un programa universal de tamizaje, aunque al igual que Estados Unidos de Norteamérica, no es obligatorio. Existen programas universales obligatorios en Alemania, Filipinas, y en implementación en Australia. En Europa, la mitad de los países tienen programas de tamizaje universal ${ }^{22}$. 
En Chile, desde el año 2005, en el contexto de las garantías explícitas en salud (GES), contamos con el programa de pesquisa de hipoacusia selectivo en el recién nacido prematuro menor de 32 semanas o de peso al nacer menor a 1.500 gramos $^{11}$. Sin embargo, este programa sólo realiza tamizaje a $0,99 \%$ de los recién nacidos vivos por año, de acuerdo a cifras del programa de seguimiento de prematuros, ya que se restringe a sólo dos factores de riesgo. Esto corresponde a 1.467 recién nacidos por año en estas condiciones ${ }^{25}$. La Encuesta de Discapacidad del año 2004 informó que la hipoacusia era la segunda causa de discapacidad en Chile. Otros resultados indican que $70,1 \%$ de las personas con hipoacusia mayores de 15 años no realizaban actividad remunerada, que $43 \%$ de las personas con hipoacusia tiene educación básica incompleta y que sólo $6 \%$ de las personas con hipoacusia ha tenido acceso a la educación superior ${ }^{26}$. De esta forma los pacientes con hipoacusias severas y profundas cuentan con pensión de invalidez o bien doble asignación familiar antes de los 18 años, siendo considerados discapacitados. Considerando que la mitad de los casos no tiene ningún factor de riesgo, hay una gran proporción de recién nacidos con hipoacusia que estarían quedando sin ser detectados.

Pese al avance local, cada vez son más los estudios y evidencias de la esfera clínica, epidemiológica y económica que apuntan a la universalidad del programa, ya que se ha logrado identificar que los programas basados en la pesquisa dirigida en la población de mayor riesgo de hipoacusia dejan una gran proporción de casos sin diagnóstico $^{4,11,27-31}$. Esta es una importante razón que justifica la implementación de programas de pesquisa que abarquen a la totalidad de los recién nacidos.

En este contexto se hace evidente que la cobertura del actual programa público chileno de pesquisa de hipoacusia, que abarca sólo a 2 de los múltiples factores de riesgo conocidos, es un conflicto de importancia significativa en salud pública por la gran carga social y económica que significa habilitar a un niño hipoacúsico no pesquisado de forma precoz.

\section{Iniciativas de tamizaje en Chile}

En Chile existen iniciativas de tamizaje diversas. En el sector público, el Hospital Dr. Sótero del Río cuenta con un programa de tamizaje universal en funcionamiento desde el año $2005^{32}$.
En Temuco, desde septiembre del mismo año se realiza detección en Puerperio y en Neonatología del Hospital Dr. Hernán Henríquez Aravena; en el Hospital Las Higueras de Talcahuano se realiza tamizaje neonatal universal desde el año 2000. Además el Hospital Barros Luco Trudeau cuenta con un programa piloto de implantes cocleares desde el año 2003, el Hospital Luis Calvo Mackenna es centro de derivación de implantes cocleares de JUNAEB, y el Hospital Naval de Viña del Mar también es centro de derivación del MINSAL.

Por otro lado, en el ámbito privado, Clínica Las Condes tiene un programa de tamizaje universal ininterrumpido desde el año 2001 y un programa de implantes cocleares y rehabilitación desde $1994^{20}$. Además, Clínica Alemana de Santiago tiene un programa establecido de tamizaje e implantes cocleares desde hace algunos años, el Hospital Clínico de la Universidad de Chile y las clínicas Santa María y Tabancura, el Hospital Militar, el Hospital Naval y el de la Pontificia Universidad Católica de Chile también tienen programas de tamizaje. Posiblemente, otras instituciones privadas, tanto en Santiago como en provincias también posean programas establecidos de tamizaje, pero no existen reportes formales de sus experiencias.

\section{Hacia un Programa de tamizaje universal e intervención precoz (PTUIP) en hipoacusia sensorioneural bilateral congénita}

Los métodos de tamizaje en salud deben cumplir una serie de requisitos (Tabla 1) que en el caso de la HSNBC son ampliamente cubiertos.

\section{Tabla 1. Condiciones necesarias para un programa de tamizaje*}

1. Métodos de pesquisa con elevada sensibilidad

2. Disponibilidad de pruebas de confirmación diagnóstica con elevada especificidad

3. Costos asociados aceptables

4. Intervenciones efectivas de tratamiento

5. Intervenciones efectivas de rehabilitación

6. Conocimiento del pronóstico e historia natural de la enfermedad en caso de intervenir o no

*Wilson J, Jungner G. Principles and Practice of Screening for Disease. Public Health Papers, № 34. Geneva, Switzerland: World Health Organization; 1968. 
Cobra gran relevancia el hecho de que ésta no es detectable de manera precoz por otros parámetros clínicos ${ }^{10}$. Por esta razón, adquiere importancia la implementación de PTUIP en hipoacusia neona$\mathrm{tal}^{22}$. Siguiendo estos principios, se recomienda que la detección de hipoacusia no sea realizada con estrategias basadas en poblaciones de riesgo, debido a su limitada efectividad ${ }^{33}$ ya que el tamizaje universal permite una mejor derivación, diagnóstico y oportunidad de acceso a tratamiento ${ }^{13}$.

\section{Métodos de pesquisa}

En relación a los métodos de tamizaje, se utilizan fundamentalmente dos: otoemisiones acústicas (OEA) y potenciales evocados auditivos de tronco abreviados (PEAT-A). Ambos son exámenes fisiológicos, no invasivos, y pueden ser realizados junto a la cama del recién nacido ${ }^{34}$. Las OEA evalúan la vía auditiva desde el conducto auditivo externo hasta el oído interno, y se encuentran presentes en $99 \%$ de los oídos sanos. Los PEAT-A evalúan la vía auditiva desde el conducto auditivo externo hasta el tronco cerebral ${ }^{34}$. En la mayoría de los programas de tamizaje universal se ha implementado una estrategia en dos pasos, por ser costo efectiva y más certera ${ }^{34}$. Lo habitual es la realización de OEA en todos los niños como primer paso, y la realización de PEAT-A en los niños que no pasen las OEA, y en casos de niños con factores de riesgo conocidos para hipoacusia ${ }^{35}$.

\section{Aspectos económicos}

Entre los factores que actúan como barreras para la implementación de programas de salud, y en los PTUIP en particular, se incluyen la falta de financiamiento, equipos de tamizaje y diagnósticos, falta de conciencia de la opinión pública sobre el tema (awareness), y falta de personal capacitado suficiente ${ }^{16}$.

En relación a costo-efectividad de un programa de tamizaje universal, se debe considerar que los costos de tener personas con hipoacusia son considerablemente altos para la sociedad. Se han realizado estudios que comparan los costos que significa tener un discapacitado auditivo a lo largo de su vida, con sus implicancias académicas y productivas, versus un individuo socialmente activo. Los PTUIP han significado ahorro en millones a los Estados, y al sector salud un ahorro de prestaciones sanitarias a lo largo de toda la vida ${ }^{36}$.

En Inglaterra se estimaron gastos adicionales de cerca de USD 14.000 en niños hipoacúsicos versus niños sanos para el período entre el nacimiento y los 9 años, que aumentan en la medida que existen menores habilidades de lenguaje oral ${ }^{36}$. En Canadá un reporte informó que el costo de implementación total de un programa de pesquisa universal de hipoacusia fue de USD 5.200.000, pero con un beneficio en términos de ahorro por año de USD 1.700.000 principalmente por concepto de ahorro educacional y prestaciones sanitarias ${ }^{34}$.

Las fuentes de financiamiento de los programas de tamizaje tienen diferentes modalidades. Existen aportes del Estado o de las familias con su aporte de bolsillo, o bien modalidades de financiamiento mixto por ambas partes de acuerdo al centro de atención específico. En países como Argentina, India, Serbia y parcialmente Brasil, el Estado es la principal fuente de financiamiento en hospitales públicos. En China y Estados Unidos de Norteamérica, son las familias las que deben hacer el mayor esfuerzo financiero ${ }^{16,22}$. En el caso chileno a nivel público los programas de pesquisa y la implementación son financiados principalmente por el Ministerio de Salud. En el sector privado son las familias las encargadas de financiar la pesquisa, diagnóstico y tratamiento de la HSNBC.

\section{Implementación del programa}

Las diferencias entre los sistemas de salud de los distintos países que han implementado programas de tamizaje, generan diferentes planteamientos respecto a la implementación. Pese a ello, la OMS promueve los factores que estarían asociados con mejores resultados en los programas de tamizaje de hipoacusia (Tabla 2$)^{22}$.

Se debe tener en consideración que el programa de tamizaje debe estar vinculado a un sistema efectivo y oportuno de diagnóstico, intervención, y apoyo familiar, que son los siguientes pasos de la cadena $^{22}$. Además, existe evidencia respecto a que los niños con hipoacusia son mejor manejados por equipos multidisciplinarios ${ }^{34}$.

Es necesario realizar el debate respecto a los pasos para la implementación de un PTUIP. Como se mencionó, actualmente existen iniciativas de tamizaje en múltiples centros, tanto públicos como privados, vinculados o no a Universidades. Es importante rescatar todas estas experiencias al momento de plantear un eventual programa de carácter nacional.

Para avanzar hacia un PTUIP nacional, se pue- 
de lograr mediante un programa piloto universal distrital (hospitalario, servicio de salud, o regional) bien diseñado y planificado, con resultados intermedios de gestión y clínicos para evaluar la viabilidad de la política, o mediante un programa piloto de detección basado en todos los factores de riesgo conocidos, que sea monitorizado con los mismos indicadores ${ }^{37}$. En cualquier caso, no estaría recomendado implementar de inmediato un PTUIP nacional sin la experiencia previa formal de un programa piloto inicial.

Para la implementación de una política pública en el manejo de la hipoacusia, se consideran cinco etapas: sospecha de hipoacusia, diagnóstico, implementación de ayuda auditiva (que puede ser con audífonos o implante coclear), habilitación, y seguimiento ${ }^{11}$. Estas 5 etapas podrían ser consideradas como paquetes de prestaciones que pudieran ser valorizadas por los tomadores de decisiones, de manera de promover la generación de un futuro programa de política pública.

\section{Conclusiones}

Chile es uno de los países latinoamericanos con mayor ingreso nacional per cápita, y cuenta

\section{Tabla 2. Factores asociados con mejores resultados en programas de tamizaje de hipoacusia promovidos por la OMS*}

1. Programas con objetivos, roles y responsabilidades bien definidas

2. Persona a cargo con responsabilidades respecto al funcionamiento del programa

3. Protocolo de tamizaje claro, basado en circunstancias locales y socializadas con los actores relevantes

4. Monitoreo periódico para verificar la correcta ejecución del programa

5. Entrenamiento adecuado del personal que ejecute el tamizaje

6. Comunicación efectiva desde el personal hacia los padres

7. Procedimientos de aseguramiento de calidad del programa

8. Seguimiento de los casos y los resultados

*World Health Organization. Newborn and Infant Hearing screening. Current issues and guiding principles for action. Outcome of a WHO informal consultation held at WHO headquarters, Geneva, Switzerland, 09-10 November 2009. con escasos programas de pesquisa de hipoacusia. A su vez, posee características inmejorables para ampliar el programa nacional actual, no sólo en lo que se refiere a su estabilidad económica y sociopolítica, sino que posee uno de los mejores índices sanitarios del continente en lo que a atención del parto y recién nacido se refiere, tema relevante, ya que es durante la hospitalización por el parto el momento más indicado para realizar la pesquisa de hipoacusia ${ }^{16}$.

Las estrategias de tamizaje han generado descenso en la edad de identificación y diagnóstico, y descenso en la edad de inicio de implementación de terapia o habilitación ${ }^{4}$. Existen 2 revisiones sistemáticas de la literatura que apoyan el hecho que los niños que son sometidos a un programa de tamizaje universal son diagnosticados precozmente y reciben tratamiento antes ${ }^{13,38}$. Lo relevante de esto es que mientras antes se haga el diagnóstico, antes se puede intervenir, lo que es fundamental en audición ya que a mayor edad de intervención, peores resultados en términos del desarrollo de lenguaje y otras habilidades cognitivas ${ }^{4}$.

Respecto a la alternativa del tamizaje universal, desde el punto de vista ético, la convención de la OMS sobre "Derechos de las personas con discapacidades" hace énfasis en los derechos de los niños con discapacidades, específicamente en relación a que la rehabilitación debe ser promovida lo más precoz posible ${ }^{22}$. En este punto, surge un dilema ético en el sentido de que si se pretende implementar un programa de tamizaje universal de hipoacusia, se debe plantear la posibilidad que el Estado financie el tratamiento para los casos en que la familia no pueda asumirlos.

Es relevante que esta situación se esté abordando desde las políticas públicas en salud. Se requerirá un esfuerzo conjunto por múltiples equipos de trabajo que partan desde la atención primaria, las unidades obstétricas y neonatales, los equipos otorrinolaringológicos y audiológicos, la cooperación materna, entre muchos otros, ya que la tarea es compleja. Sin embargo, con el funcionamiento del GES ya se cuenta con una experiencia que puede ser facilitadora para este nuevo proceso.

En Chile este es un problema relevante, muchas veces subestimado por los medios y los tomadores de decisión, que tiene potencialmente solución, ya que existe la experticia técnica y eventualmente los recursos para concretarlo. Para esto se requiere implementar un programa de tamizaje con el 
apoyo de personal e infraestructura dedicada al programa. A su vez, se debe contar con un sistema de registro y seguimiento adecuado, y evaluaciones de costo y pronóstico de los casos detectados de manera de evaluar el verdadero impacto.

\section{Referencias}

1. Yoshinaga-Itano C, Sedey AL, Coulter DK, Mehl AL. Language of early-and later-identified children with hearing loss. Pediatrics 1998; 102 (5): 1161-71.

2. Parving A. Congenital hearing disability-epidemiology and identification: a comparison between two health authority districts. Int J Pediatr Otorhinolaryngol 1993; 27 (1): 29-46.

3. Mehra S, Eavey RD, Keamy DG, Jr. The epidemiology of hearing impairment in the United States: newborns, children, and adolescents. Otolaryngol Head Neck Surg 2009; 140 (4): 461-72.

4. Joint Committee on Infant Hearing. Year 2007 position statement: Principles and guidelines for early hearing detection and intervention programs. Pediatrics 2007; 120 (4): 898-921.

5. Kennedy CR, McCann DC, Campbell MJ, Law CM, Mullee M, Petrou S, et al. Language ability after early detection of permanent childhood hearing impairment. N Engl J Med 2006; 354 (20): 2131-41.

6. Controlled trial of universal neonatal screening for early identification of permanent childhood hearing impairment. Wessex Universal Neonatal Hearing Screening Trial Group. Lancet 1998; 352 (9145): 1957-64.

7. Holden-Pitt L, Albertorio J. Thirty years of the Annual Survey of Deaf and Hard-of-Hearing Children \& Youth: a glance over the decades. Am Ann Deaf 1998; 143 (2): 72-6.

8. Carney AE, Moeller MP. Treatment efficacy: hearing loss in children. J Speech Lang Hear Res 1998; 41 (1): S61-84.

9. Connolly JL, Carron JD, Roark SD. Universal newborn hearing screening: are we achieving the Joint Committee on Infant Hearing ( JCIH) objectives? Laryngoscope. 2005; 115 (2): 232-6.

10. Kerschner JE. Neonatal hearing screening: to do or not to do. Pediatrics Clinics of North America 2004; 51: 725 36.

11. Pittaluga E. Guía clínica: hipoacusia neurosensorial bilateral del prematuro. Gobierno de Chile, Ministerio de Salud. 2009. Citado el 20 de diciembre de 2011: Disponible en: http://www.supersalud.gob.cl/difusion/572/ articles-6447_recurso_1.pdf.

12. Alvo A, Der C, Delano P. Tamizaje universal de hipoacu- sia en el recién nacido. Rev Hosp Clin Univ Chile 2010; 21: 170-6.

13. Nelson HD, Bougatsos C, Nygren P. Universal newborn hearing screening: systematic review to update the 2001 US Preventive Services Task Force Recommendation. Pediatrics 2008; 122 (1): e266-76.

14. Francis HW, Koch ME, Wyatt JR, Niparko JK. Trends in educational placement and cost-benefit considerations in children with cochlear implants. Arch Otolaryngol Head Neck Surg 1999; 125 (5): 499-505.

15. Cheng AK, Rubin HR, Powe NR, Mellon NK, Francis HW, Niparko JK. Cost-utility analysis of the cochlear implant in children. JAMA. 2000 Aug 16; 284 (7): 850-6.

16. Gerner de García B, Gaffney C, Chacon S, Gaffney M. Overview of newborn hearing screening activities in Latin America. Rev Panam Salud Pública 2011; 29 (3): 145-52.

17. Morton CC, Nance WE. Newborn hearing screening-a silent revolution. N Engl J Med 2006; 354 (20): 2151-64.

18. Vohr B. Overview: Infants and children with hearing loss-part I. Ment Retard Dev Disabil Res Rev 2003; 9 (2): 62-4.

19. Encuesta de Caracterización Socio Económico Nacional (CASEN) 2009. Citada el 10 de enero de 2012: Disponible en: http://www.ministeriodesarrollosocial.gob.cl/ casen2009.

20. Nazar G, Goycoolea M, Godoy J, Ried E, Sierra M. Evaluación auditiva neonatal universal: Revisión de 10.000 pacientes estudiados. Rev Otorrinolaringol Cir Cabeza Cuello 2009; 69: 93-102.

21. Asociación Argentina de Logopedia Foniatría y Audiología. Consenso Argentino de intervención temprana de la hipoacusia infantil. Buenos Aires: CEIDHI. Citado el 13 de diciembre de 2011: Disponible en: http://www.ceidhi. org.ar/novedades.php.

22. World Health Organization. Newborn and Infant Hearing screening. Current issues and guiding principles for action. Outcome of a WHO informal consultation held at WHO headquarters, Geneva, Switzerland, 09-10 November 2009. Citado el 25 de febrero de 2012: Disponible en: http://www.isa-audiology.org/pdf/Newborn_and_Infant_Hearing_Screening.pdf.

23. Early Identification of Hearing Impairment in Infants and Young Children. NIH Consens Statement 1993; 11 (1): $1-24$.

24. Joint Committee on Infant Hearing (JCIH): Joint Committee on Infant Hearing Year 2000 Position Statement: Principles and guidelines for early hearing detection and intervention programmes. Pediatrics 2000; 106: 798-817.

25. Recién Nacidos con menos de 32 semanas de edad gestacional. Sistema Nacional de Servicios de Salud de 
Chile. Quinquenio 2000-2004. Citado el 13 de diciembre de 2011: Disponible en: http://www.prematuros.cl/ webmarzo08/InformeRN.pdf.

26. Primera Encuesta de Discapacidad, ENDISC, FONADIS 2004. Citado el 20 de febrero de 2012: Disponible en: http://www.ine.cl/canales/chile_estadistico/encuestas_discapacidad/pdf/apartadotematicodeficienciaauditivaytrastornosseverosdelacom.pdf.

27. Keren R, Helfand M, Homer C, McPhillips H, Lieu TA. Projected cost-effectiveness of statewide universal newborn hearing screening. Pediatrics 2002; 110 (5): 855-64.

28. Gorga MP, Neely ST. Cost-effectiveness and test-performance factors in relation to universal newborn hearing screening. Ment Retard Dev Disabil Res Rev 2003; 9 (2): 103-8.

29. Wrightson AS. Universal newborn hearing screening. Am Fam Physician 2007 May 1; 75 (9): 1349-52.

30. Erenberg A, Lemons J, Sia C, Trunkel D, Ziring P. Newborn and infant hearing loss: detection and intervention. American Academy of Pediatrics. Task Force on Newborn and Infant Hearing, 1998-1999. Pediatrics 1999; 103 (2): 527-30.

31. Iñíguez R, Cevo T, Fernández F, Godoy C, Iñíguez R. Detección precoz de pérdida auditiva en niños con factores de riesgo. Rev Otorrinolaringol Cir Cabeza Cuello 2004;
64: 99-104.

32. Godoy C, Bustamante L. Evaluación de la fase de screening auditivo en menores con factores de riesgo. Rev Otorrinolaringol Cir Cabeza Cuello 2006; 66: 103-6.

33. Olusanya BO. Making targeted screening for infant hearing loss an effective option in less developed countries. Int J Pediatr Otorhinolaryngol 2011; 75: 316-21.

34. Patel H, Feldman M. Universal newborn hearing screening. Paediatrics \& Child Health 2011; 16 (5).

35. McManus MA, Levtov R, White KR, Forsman I, Foust T, Thompson M. Medicaid Reimbursement of Hearing Services for Infants and Young Children. Pediatrics 2010; 126: S34.

36. Schroeder L, Petrou S, Kennedy C, McCann D, Law C, Watkin PM, et al. The economic costs of congenital bilateral permanent childhood hearing impairment. Pediatrics 2006; 117 (4): 1101-12.

37. Grill E, Hessel F, Siebert U, Schnell-Inderst P, Kunze S, Nickisch A, et al. Comparing the clinical effectiveness of different new-born hearing screening strategies. A decision analysis. BMC Public Health 2005 Jan 31; 5: 12.

38. Bielecki I, Horbulewicz A, Wolan T. Risk factors associated with hearing loss in infants: an analysis of 5282 referred neonates. Int J Pediatr Otorhinolaryngol 2011; 75 (7): 925-30. 